\title{
Design and Implementation of intelligent storage system
}

\author{
Ke-Ran Bai ${ }^{\mathrm{a}}$, Yi-Xian Chen ${ }^{\mathrm{b}^{*}}$, Yang-Yu-Zi He ${ }^{\mathrm{c}}$, Jun-Zheng Zheng, Guo-Liang \\ Wu
}

College of Electrical and Information Engineering, Southwest Minzu University, Chengdu, China a2049591243@qq.com, ${ }^{\mathrm{b}}$ cyxian@hotmail.com, ${ }^{\circ}$ 527657192@qq.com

Keywords: Intelligent storage; Sensor; Handling robots; host computer

Abstract. In order to realize the automated warehousing information management, a storage information management system based on patrol robot technology was designed and developed. The robot's core controller uses a STM32F104 chip produced by ST Company. The position information of the robot is obtained through an external auxiliary detection sensor and an encoder. The system outputs the location information of the warehouse items stored in the database to the host computer, and then the host computer sends the information to the handling robot by wireless to achieve the accurate and rapid handling of the warehouse goods.

\section{Introduction}

With the rapid development of e-commerce, the logistics business has risen sharply and the cost of land and manpower has risen rapidly, the industry has been looking for intelligent logistics equipment that can save warehouse space and improve logistics efficiency. In addition to intelligent management facilities, but also need to have to replace the artificial handling, handling, packaging equipment, including intelligent storage robot is a typical representative.

Can be seen in the Internet economy and the trend of Internet industry, warehousing and logistics industry is ushering in unprecedented opportunities for development and new patterns of change ${ }^{[1]}$. The application of robotics has become an important measure of competition and future development among enterprises. As new robotic technologies continue to emerge, more and more intelligent robot applications will emerge in other areas of logistics. Warehousing logistics robots will have a far-reaching impact on the logistics operation mode and the entire logistics system transformation.

\section{Design of robot hardware system and mechanical structure}

Overview of the overall structure of the robot. The main controller of the robot hardware system is STM32F103, and Robot use the McNum wheel to support the robot's steering and movement. At the same time, the speed control module, the power management module, the image information acquisition module and the DC motor drive module are integrated. Mechanical structure part of the self-designed mechanical claw used to insert the target goods. Electric screwdriver equipped with screw bearings. The robot is shown in Fig.1.

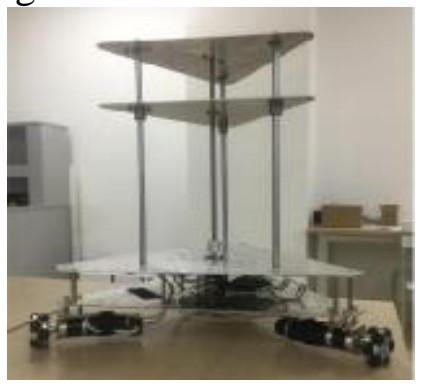

Figure 1. Overall mechanical structure of the robot 


\section{Robot software system design}

\section{Control the movement of the body design.}

Taking into account the robot path planning and accessing to existing information. We have learned that the existing methods of robot path planning mainly include: Visibility Graph, Free Space, Grid, optimal control method, Topological method, neural network method, etc. The grid method ${ }^{[2][3]}$ is the most widely used path planning method.

In this paper, the author thinks that this special geographical location of warehouse has a large space, flexible range of activities, and moving obstacles less and fixed. If we want to use the grid method of robot path planning, then the need to collect information beyond the existing rapid processing of the chip, resulting in time delay and some unnecessary trouble.

In addition, the light in the warehouse can be adjusted, so that some of the finer details of the images taken by the camera will not be mistaken or even misjudged because of light restrictions. We decided to make a special arrangement for the warehouse site, establish a three-dimensional coordinate system with the warehouse floor level and the corresponding shelf, and perform three-dimensional coordinate coding on the site location and the commodity location. The coordinate information contains the position of the product in the warehouse and the position of the product on the shelf.

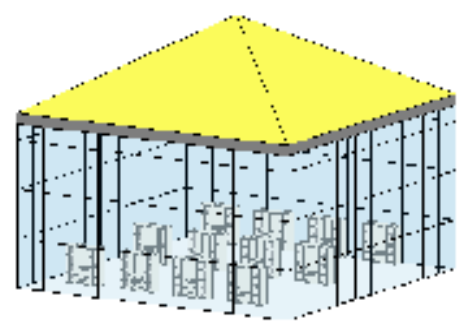

Figure 2. Warehouse Site Layout Diagram

The camera shot images processed, the processed information is converted into control signals in order to control the robot's caster movement direction and speed of movement. At the same time, the robot's chassis is supplemented by the audience positioning module, so as to correct the position and posture of the robot so that the robot can accurately reach the designated position.

\section{Mobile Parts Software Operation Flow.}

Robot software design consists of two main components:

It identifies the image captured by the camera, removes the noise caused by the light in the image to accurately and extracts the black guideline in the driving path. According to the difference between the color of the black guide line and the laying site, the effect on the captured picture of the camera is that there is a big difference between the edge of the black guide line and the background. That is to say, so the edge extraction algorithm is adopted when the image is processed.

According to the site information acquired by the camera acquisition and processing and vehicle speed information obtained by the vehicle speed detection module for kinematic analysis, combined with the control algorithm, it outputs the steering control of the omnidirectional wheel and the speed control of the motor, so that the robot can drive fast along the black guiding line. The software process is shown in Fig.3. 


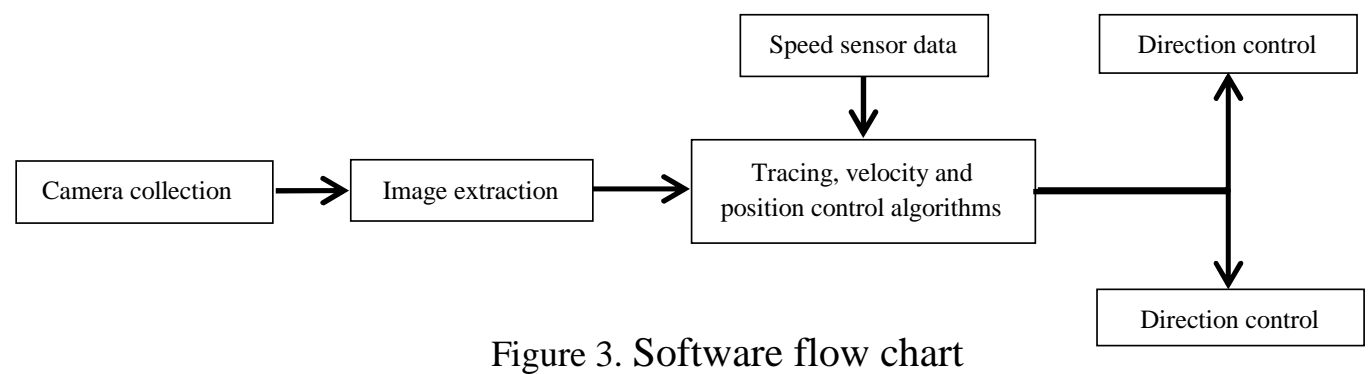

\section{Site guideline extraction and identification.}

Figure 3. Software flow chart

Venue information collection. Taking into account the robots arranged in a special place such as the warehouse scene, the light in the warehouse can be specially processed, which can reduce the impact of light on the camera, thus reducing the noise removal processing, thereby increasing the utilization of the control chip.

Lead recognition. Extract the part of the image captured by the camera that reflects the track information. This is a process of image segmentation. Based on the color of the site and the black lead laid, we use a linearization method that has the advantages of simplicity and convenience to meet user requirements for data processing.

\section{Kinematics analysis.}

The basis of robot control is kinematic analysis. The kinematics analysis can be used to get the rule of the variation of various parameters and their relationship with each other during the motion of the robot, and then verify with the controller through the program. Before making motion modeling, in order to simplify the mathematical model of kinematics, we made the following idealized assumptions:

I Omnidirectional wheel does not slip with the ground, while the ground has enough friction;

I The center of the motor axis is the center of the chassis;

I Between the wheels is absolutely $120^{\circ}$ installation;

I It is assumed that the body coordinate system coincides with the geographic coordinate system.

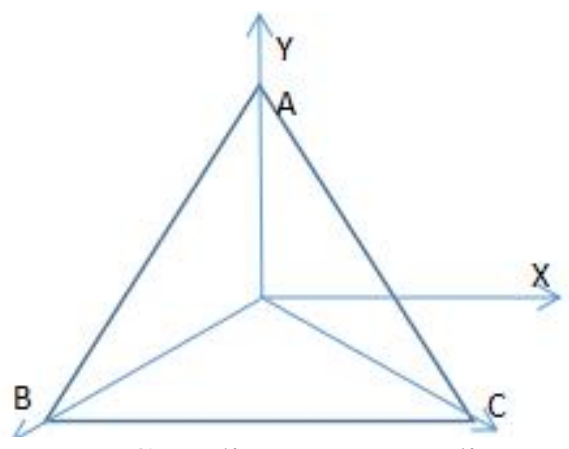

Figure 4. Coordinate system diagram

Speed decomposition can get the following formula

$$
\begin{aligned}
& V_{A}=V_{X}+L \omega \\
& V_{B}=-\operatorname{Sin} 30^{\circ} V_{X}-\operatorname{Sin} 60^{\circ} V_{Y}+L \omega \\
& V_{c}=-\operatorname{Cos} 60^{\circ} V_{X}-\operatorname{Sin} 60^{\circ} V_{Y}+L \omega
\end{aligned}
$$

Then:

$$
\begin{aligned}
& V_{A}=V_{X}+L \omega \\
& V_{B}=-\frac{1}{2} V_{X}+\frac{\sqrt{3}}{2} V_{Y}+L \omega \\
& V_{C}=-\frac{1}{2} V_{X}-\frac{\sqrt{3}}{2} V_{Y}+L \omega
\end{aligned}
$$

$\omega$ is robot angular velocity, $\mathrm{L}$ is the distance between the center of the omnidirectional wheel and the center of the chassis, $V_{A}, V_{B}, V_{C}$ are the speed of three wheels respectively, $V_{X}, V_{Y}$ is the robot's $\mathrm{X}, \mathrm{Y}$ direction of the speed of movement. 


\section{Database and computer program design}

\section{Design of the overall framework of the database}

The author of this paper designs a warehouse data information management system to adapt to the development of scientific and information zed warehouse.

1. To complete the input of various information in the warehouse, such as the storage of goods, a library, the coordinates of the goods, the confirmation of order information;

2. To modify and maintain a variety of information in the warehouse;

3. To achieve real-time query statistics of various goods information in the warehouse;

4. To realize the warehouse goods order information record.

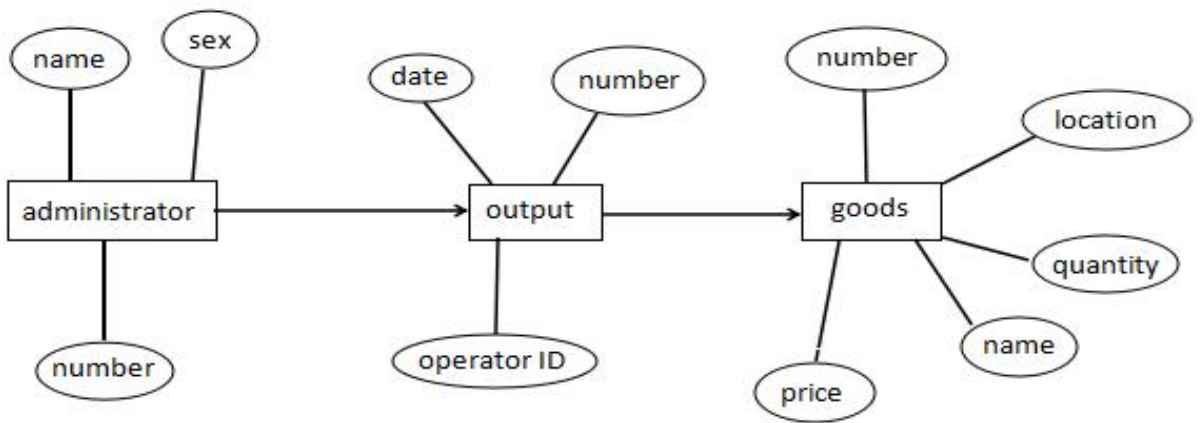

Fig.5Database part of the simple E-R diagram

We built the table using the MySQL language on the Navicat for MySQL development platform.

PC programming

PC's main function is to provide users with a better man-machine communication interface, the storage of warehouse information in the database of commodity information for simple processing, generating a graph, and presented in the form of statements. This has greatly promoted the user's preparation and decision-making of the product reserve in the warehouse.

When testing the system at work, it is necessary to send commands such as picking up, as well as the data returned by the lower computer. pc using single-threaded programming, pc cannot meet the real-time two tasks at the same time, we need a main thread to complete the user interface to the operation, while creating a secondary thread to complete the data receiving operation to meet the fluency of the system operation and real-time ${ }^{[4]}$.

\section{Conclusions}

The entire system integrates robot control, PC transmission, database content of the three major aspects of the professional knowledge and skills are more extensive requirements. After debugging and further improvement of the control program, the upper computer part can accurately communicate the information with the robot. In the aspect of image processing algorithm, the Robert operator currently used in the image processing is not very smooth edges, the need to continue to improve.

\section{Acknowledgements}

This work was financially supported by the innovative training program for college students, Southwest Minzu University (201710656009).

\section{References}

[1] China Society of Logistics, Analysis of the development prospect of China's logistics and warehousing industry in 2016

[2] Wang Yanping, Research on Path Planning Methods of Mobile Robot [J]. Computer \& Telecommunication, 2009, 30(1): 66-68. 
[3] YU Hong-bin, LI Xiao-an, Fast Path Planning Based on Grid Model of Robot [J], Microelectronics \& Computer, 2005,22(6):98-100.

[4] WU Xian-liang, LIU Chun-Sheng, Programming of Serial Communication Design and Implement Based on Multi-thread [J], CONTROL ENGINEERING OF CHINA,2004(02):171-174.

[5] MA Xiang, Research on Intelligent Logistics Control System Based on ZigBee Wireless Network [J], Journal of Harbin University of Science and Technology,2016,21(06):67-72.

[6] YU Xiaoqing, ZHAO Weijie, LI Xiaoqing, Computer communication and database management system based on C\#.Net in public umbrellas rental system [J], Industrial Instrumentation \& Automation,2013(05):70-72+113.

[7] Widodo Budiharto, Ari Santoso, Djoko Purwanto, Achmad Jazidie. A Method for Path Planning Strategy and Navigation of Service Robot[J]. Paladyn, Journal of Behavioral Robotics,2011,2(2). [8] Azani Cempaka Sari, Anita Rahayu, Widodo Budiharto. Developing Information System of Attendance and Facebook Status for Binus University's Lecturer Using Raspberry Pi Architecture[J]. Procedia Computer Science,2015,59. 